\title{
Direct Measurement of HH2-HH1 Intersubband Lifetimes in SiGe Quantum Cascade Structures
}

\author{
P. Rauter ${ }^{1}$, T. Fromherz ${ }^{1}$, G. Bauer ${ }^{1}$, N.Q.Vinh ${ }^{2}$, P.J. Phillips ${ }^{3}$, C.R. Pidgeon ${ }^{3}$, B.N. Murdin ${ }^{4}$, \\ L. Diehl ${ }^{6}$, G. Dehlinger ${ }^{5}$, D. Grützmacher ${ }^{5}$ \\ ${ }^{1}$ Institute for Semiconductor and Solid State Physics, Johannes-Kepler-University Linz, Austria \\ ${ }^{2}$ FOM Institute for Plasma Physics Rijnhuizen, Nieuwegein, Netherlands \\ ${ }^{3}$ Heriot-Watt University, Edinburgh, UK \\ ${ }^{4}$ University of Surrey, Guildford, UK \\ ${ }^{5}$ Paul Scherrer Institut, Villingen, Switzerland \\ ${ }^{6}$ University of Princeton, USA
}

\section{Introduction}

Driven by the strong need for Si-based optoelectronic devices for a wide range of applications considerable endeavors have been made to develop a laser in this material system. Silicon is an indirect semiconductor and therefore cannot be used for direct optical transitions over the bandgap. However, the concept of infrared emitters based on quantum cascade heterostructures, which is very successfully applied to III-V material systems, constitutes a promising approach towards a SiGe infrared laser. But while infrared electroluminescence (EL) of various wavelengths has been demonstrated for p-type SiGe quantum cascade structures [1-3], lasing has yet to be achieved.

One of the key issues for the achievement of lasing is the build up of population inversion, which is essentially dependent on the excited state's lifetime. A direct experimental determination of these lifetimes is, therefore, of high interest. The direct measurement of the excited state lifetimes by pump-probe transmission experiments yields clear results of $>20 \mathrm{ps}$ for QW transitions in the $\mathrm{THz}$ region (i.e. below the optical phonon energy) [4]. However, for transition energies in excess of the optical phonon energy, the effective depopulation of the excited states by deformation potential scattering results in extremely short lifetimes. Pump-probe transmission measurements revealed lifetimes of approximately $210 \mathrm{fs}$ as reported by Kaindl et al.[5]. In their work, a contribution due to hole heating was subtracted from the observed transmission transient in order to obtain this value.

\section{Measurements}

In this work, we report a direct measurement of the excited hole lifetime for transition energies larger than the optical phonon energy. We have performed

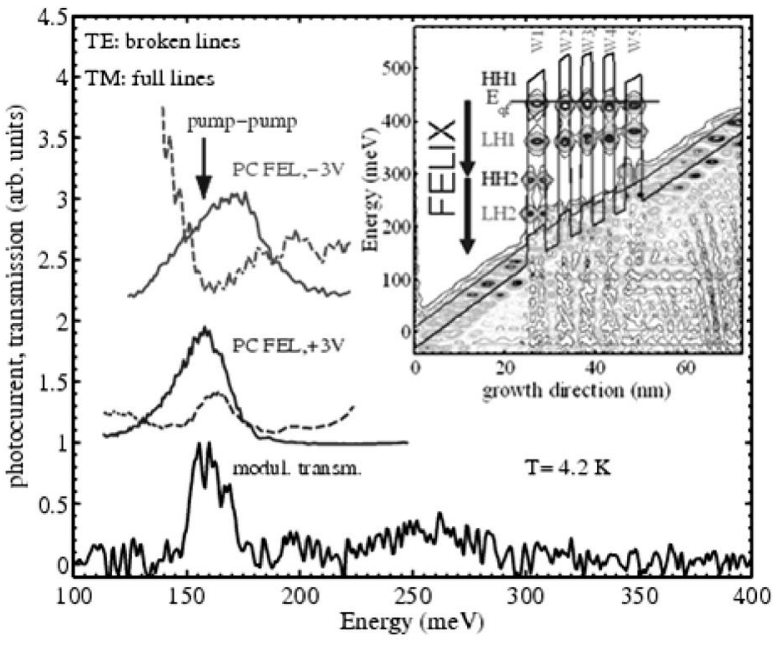

Fig. 1. Characterization of the HH1-HH2 transition energy. Both bias-modulated transmission measurements and photocurrent spectra using two-photon-absorption in TM polarization reveal a value of $160 \mathrm{meV}$, which is consistent with simulation results. Selection rules imply the measured absence of a photocurrent peak in TE polarization. The inset shows the simulated valence band edges at $\mathrm{V}=-3 \mathrm{~V}$ together with the squared wave functions at their eigenenergies. The arrows indicate the transitions HH1-HH2 and HH2-continuum at a FELIX wavelength of $7.9 \mu \mathrm{m}$.

photo-current pump-pump measurements using the free electron laser FELIX at the FOM, Nieuwegein, on a similar sample for that HH1-HH2 EL has been demonstrated [1]. FELIX provides micropulses of sub-picosecond duration and peak powers of up to 100 MW. For our experiment, the FEL wavelength was tuned into resonance with the $\mathrm{HH} 1-\mathrm{HH} 2$ transition of the deepest quantum well of the cascade $(7.9 \mu \mathrm{m})$. The energy of this transition was pre-characterized by voltage-pulsed absorption measurements on the 
structure using a FTIR spectrometer. In addition at FELIX the photocurrent through the sample was measured as a function of the laser wavelength for different polarisations. (Fig. 1)

For the determination of the HH2-HH1 relaxation time, the photocurrent through the sample was measured as a function of the delay between two FELIX micropulses, the polarizations of which differed by $90^{\circ}$. Depending on its polarization, the first laser pulse populates the confined $\mathrm{HH} 2$ state, while the second excites carriers from this state into the continuum, where charge transport is possible. The advantage of measuring the photocurrent instead of the transmission through the sample lies in its much higher sensitivity. In addition, the discrimination between pump and probe beams which is essential for pump-probe transmission experiments and is especially tedious for waveguide geometry, is not necessary in the PC setup.

\section{Results}

As the HH1-HH2 transition is only allowed in TM polarization, the gained signal curves show a pronounced asymmetry with respect to the sign of the delay between the TM and TE pulses. The fast decay observed in case the TE pulse arrives before the TM pulse (positive delay times in Fig. 2) can be associated with the temporal overlap of the two pulses and thus indicates the time resolution of our experiments. The longer decay for "TM first" originates from additional HH2-continuum transitions excited by the TE pulse out of the $\mathrm{HH} 2$ population established by the TM pulse. According to this interpretation, the observed decay directly monitors the relaxation of the non-equilibrium $\mathrm{HH} 2$ population. Measurements for different applied biases reveal a bias-independent HH2-HH1 decay time of about 550 fs. (Fig. 2)

\section{Conclusions}

We can report the first direct measurement of the $\mathrm{HH} 2-\mathrm{HH} 1$ relaxation time for a SiGe QW with transition energy above the optical phonon frequency. The experiments have been performed for an active, electrically biased SiGe structure, and thus our results constitute a key parameter for the design and dynamic simulation of $\mathrm{SiGe}$ quantum cascade laser structures. Figure 2 presents the 'as-measured' photocurrent signal data, from which only a virtually constant background has been subtracted for the 'TM first' and 'TE first' cases. On the timescale of our experiment, a exponential $\mathrm{HH} 2-\mathrm{HH} 1$ decay with a single relaxation time significantly larger $(\sim 550 \mathrm{fs})$ than the time

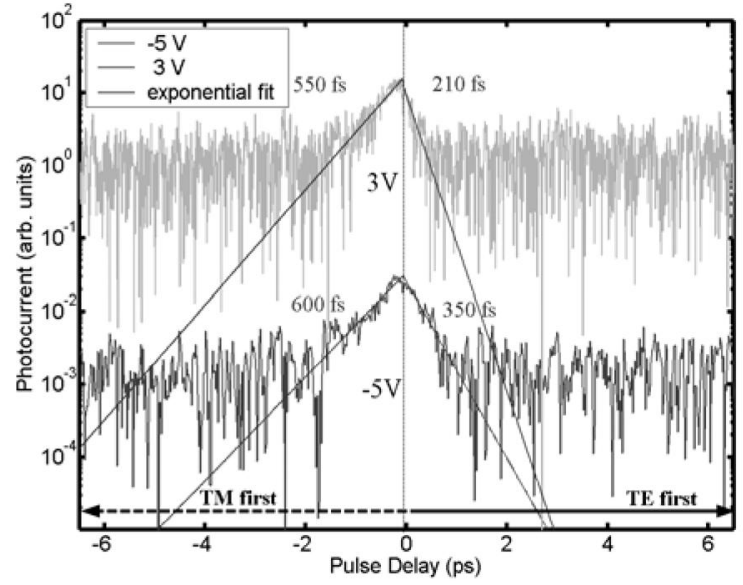

Fig. 2. Pump-pump photocurrent measurement results at the HH1-HH2 transition energy $(7.9 \mu \mathrm{m})$ for different bias voltages. The traces have been offset for clarity. The figures indicate the directly fitted exponential decay times for TM first and TE first. Since HH1-HH2 transitions are not allowed in TE polarization, the short decay constants observed for 'TE first' correspond to the temporal overlap of the laser-micropulses and thus indicate the time resolution of the experiment. The longer decay times for 'TM first' result from additional HH2-continuum transitions excited by the TE out of the TM pulse induced non-equilibrium HH2 population. Thus, the observed decay time is equal to the HH2-HH1 relaxation time.

resolution of the experiment is observed when the sample is excited by the TM pulse first. In addition the technique provides a path to the measurement of dynamics in quantum cascade structures under operating electrical injection conditions.

\section{Acknowledgements}

We gratefully acknowledge the support by the Stichting voor Fundamenteel Onderzoek der Materie (FOM) in providing the required beam time on FELIX and highly appreciate the skilful assistance by the FELIX staff. This work was supported by the European Community Research Infrastructure Action under the FP6 "Structuring the European Research Area" Programme through the Integrated Infrastructure Initiative "Integrating Activity on Synchrotron and Free Electron Laser Science", by the FWF SFB025 (IRON) and by the EC program SHINE.

\section{References}

[1] G. Dehlinger et al., Science 270, 2277 (2000),

[2] I. Bormann et al., Applied Physics Letters 80(13), 2260 (2002)

[3] S.A. Lynch et al., Material Science and Engineering B 89, $10(2002)$

[4] R.W. Kelsall et al.,Physical Review B 71, 115326 (2005)

[5] R.A. Kaindl et al., Physical Review Letters 86, 1122 (2001) 